\title{
Advances in the Micro-Hole \& Strip Plate gaseous detector
}

\author{
J.M. Maia ${ }^{\mathrm{a}, \mathrm{b}, \mathrm{c}, *}$, J.F.C.A. Veloso ${ }^{\mathrm{a}}$, J.M.F. dos Santos ${ }^{\mathrm{a}}$, A. Breskin ${ }^{\mathrm{c}}$, R. Chechik ${ }^{\mathrm{c}}$, \\ D. Mörmann ${ }^{\mathrm{c}}$ \\ ${ }^{a}$ Physics Department, University of Coimbra, P-3004-516 Coimbra, Portugal \\ ${ }^{\mathrm{b}}$ Physics Department, University of Beira Interior, P-6200-001 Covilhã, Portugal \\ ${ }^{\mathrm{c}}$ Department of Particle Physics, The Weizmann Institute of Science, 76100 Rehovot, Israel
}

\begin{abstract}
We report on the performance of a new gaseous electron multiplier: the Micro-Hole \& Strip Plate (MHSP). It consists of two independent charge-amplification stages in a single, double-sided micro-structured electrode, deposited on a thin insulating substrate. Charge gains in excess of $10^{3}$ were obtained in a MHSP operated with soft X-rays in $\mathrm{Ar} / \mathrm{CO}_{2}(70 /$ 30). We present the results of a systematic study of the MHSP properties and those of a double-stage GEM + MHSP multiplier. Applications to gaseous photomultipliers are discussed.
\end{abstract}

(C) 2003 Elsevier Science B.V. All rights reserved.

PACS: 29.40.-n; 29.40.Cs; $85.60 . \mathrm{Gz}$

Keywords: Micro-strip gas counter; Gas electron multiplier; Micro-Hole \& Strip Plate gas detector; Photosensor

\section{Introduction}

The Micro-Hole \& Strip Plate (MHSP) $[1,2]$ is a new gaseous multiplier; it was conceived following the demonstration that a micro-strip gas counter (MSGC) [3] preceded by a gas electron multiplier (GEM) [4] pre-amplifying element has better stability compared to MSGC alone [5]. The MHSP combines the characteristics of both GEM and MSGC in a single, double-sided element. It consists of two independent charge-amplification stages micro-patterned on a single thin insulating plate, metallized on both faces, as shown in Fig. 1:

\footnotetext{
*Corresponding author. Department of Particle Physics, The Weizmann Institute of Science, 76100 Rehovot, Israel. Tel.: +972-8934-2082; fax: + 972-8934-2611.

E-mail address: jmaia@gian.fis.uc.pt (J.M. Maia).
}

the first stage consists of holes, operating as a GEM, and the second stage consists of a MSGClike structure printed on the bottom face of the insulating plate. The holes are located within the cathode strips while the anode strips run between them. An electric field is established within the holes by a potential difference between the top face and the cathode strips. The fields above and below the MHSP control the charge transport into the holes and towards the anode strips, and are defined by additional electrodes (e.g. meshes). Electrons deposited by radiation in the gas volume above the MHSP, or photoproduced on a photocathode, are focused into the holes, whereby an avalanche occurs under the high electric field; the avalanche electrons are further multiplied on the anode strips, where the final charge is collected (see Fig. 2a). The MHSP can operate as a 


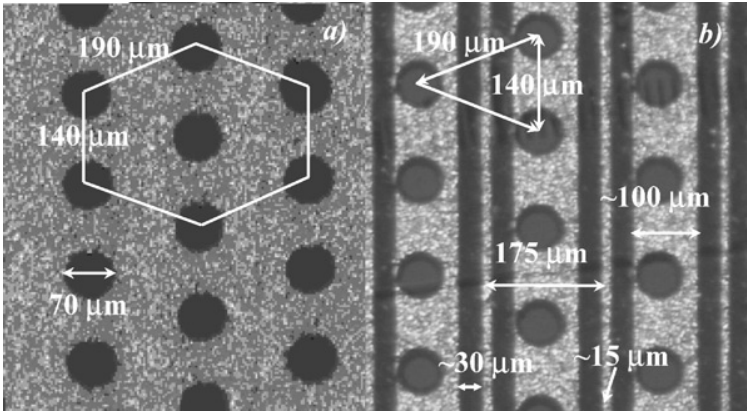

Fig. 1. Photomicrographs of the MHSP geometry: (a) top-side, (b) MS-bottom side.

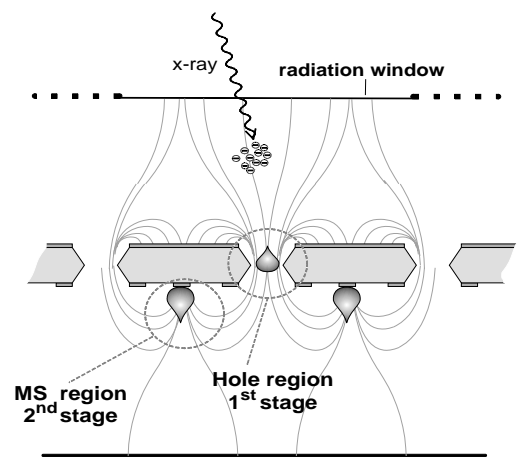

(a)

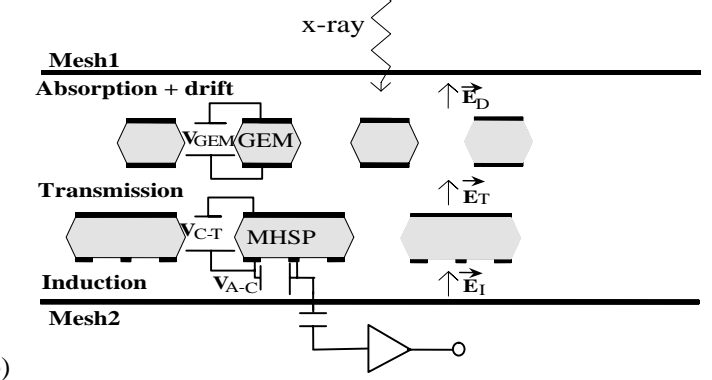

Fig. 2. A schematic of MHSP based detectors: (a) MHSP, (b) MHSP + GEM.

stand-alone multiplier, for large initial deposited charges; for small initial charge and for single electrons, it could be preceded by a preamplifying element, e.g. a GEM (Fig. 2b).

Secondary avalanche-induced photon- and ionfeedback effects often limit multiplication in gas avalanche detectors. The problem is particularly important in noble-gas mixtures, which are used in gas proportional scintillation counters [6] and in gas photomultipliers [7]. In both cases, the particular structure of the MHSP, where the final avalanche is screened by the multiplier's substrate and where part of the ions are trapped on the cathode strips, may present a solution for both feedback problems.

\section{Experimental setup}

The detector under study, shown in Fig. 2b, is assembled in a stainless-steel chamber and consists of a MHSP foil preceded by a standard GEM foil; both are enclosed between two stainless-steel meshes. The detector has a $2.5-\mathrm{mm}$ radiation absorption/electron drift region, a 2.5-mm electron transmission region and a $2.0-\mathrm{mm}$ charge induction region. Both CERN-made micro-pattern multipliers have the same Kapton and copper thickness of 50 and $5 \mu \mathrm{m}$, respectively. The MHSPs (Fig. 1) have bi-conical holes of diameter $50 \mu \mathrm{m}$ in the Kapton and $70 \mu \mathrm{m}$ in the copper, arranged in an hexagonal lattice, (two edges of $140 \mu \mathrm{m}$ and four edges of $190 \mu \mathrm{m}$ ), with an optical transparency of about $8 \%$. A micro-strip (MS) pattern of $175 \mu \mathrm{m}$ pitch with anodes and cathodes widths of 15 and $100 \mu \mathrm{m}$, respectively, is printed on the bottom-side of the Kapton foil. The anodecathode gap is $30 \mu \mathrm{m}$. The GEM has a standard hexagonal layout, of $140 \mu \mathrm{m}$ edges, with holes of 50 and $70 \mu \mathrm{m}$ in the Kapton and copper, respectively. Both multipliers have similar active area of $2.8 \times 2.8 \mathrm{~cm}^{2}$. An $\mathrm{Ar}+30 \% \mathrm{CO}_{2}$ non-flammable gas mixture at $p=760$ Torr was used in flowmode. A 1-mm diameter collimated $5.9 \mathrm{keV}$ X-ray beam from a ${ }^{55} \mathrm{Fe}$ source was used in this work. Xrays absorbed in the absorption region produce a primary cloud of about 220 electrons; these drift to the GEM holes under an appropriate drift field, $E_{\mathrm{D}}$, chosen to minimize electron losses to the top side of the GEM. Following a first multiplication in the GEM the avalanche electrons are transmitted to the MHSP holes under the transmission field, $E_{\mathrm{T}}$; its value is chosen to minimize electron losses to the bottom and top side of the GEM and the MHSP, respectively. At the MHSP, two-step multiplication occurs in the holes and on the MS 
anodes. X-rays absorbed in the transmission region (see Fig. 2b), give rise to electron clouds that experience only the two-stage multiplication of the MHSP, permitting to determine the effective gain of the MHSP alone.

All detector electrodes were independently polarized; the values for drift, transmission, and induction fields were kept constant at 1.2, 2.0 and $\sim 0.0 \mathrm{kV} / \mathrm{cm}$, respectively.

The performance of the MHSP + GEM detector was compared to that of a standard double-GEM, assembled in the same chamber and operating under the same conditions.

For detector gain studies, the collected charge from the bottom GEM electrode (in double GEM), or from the anode strips of the MHSP (in MHSP or in MHSP + GEM) was processed by a charge-sensitive preamplifier followed by a linear amplifier; the pulses were further processed by a multichannel analyser. The electronic chain was calibrated to allow for absolute gain determination. For peak-amplitude and energy resolution determination pulse-height distributions were fitted to a Gaussian, superimposed on a linear background.

Current signals were processed with two types of fast current amplifiers: the VV44-MPI Heidelberg with a rise-time of $9 \mathrm{~ns}$ and a gain of 170 provides both electron and ion components of the signal, while the VT110CH4-ESN, with a rise-time of $0.5 \mathrm{~ns}$, provides the fast component induced by electrons. These signals were recorded with a $500 \mathrm{MHz}$ digital oscilloscope Tektronix TDS 3052.

\section{Results and discussion}

In Fig. 3 we present the gain-voltage characteristics of the MHSP, MHSP + GEM and a doubleGEM, measured in $\mathrm{Ar}+30 \% \mathrm{CO}_{2}$ mixture. The first two were measured with a fixed potential difference across the MHSP holes, $V_{\mathrm{C}-\mathrm{T}}=400 \mathrm{~V}$, at two values of $V_{\mathrm{GEM}}$, and are presented as a function of the anode-to-cathode voltage, $V_{\mathrm{A}-\mathrm{C}}$; the last was measured with a fixed value of $V_{\mathrm{GEM} 1}=500 \mathrm{~V}$, and is given as a function of $V_{\text {GEM2}}$. The MHSP gain curve could be measured directly, as explained above, from the X-rays

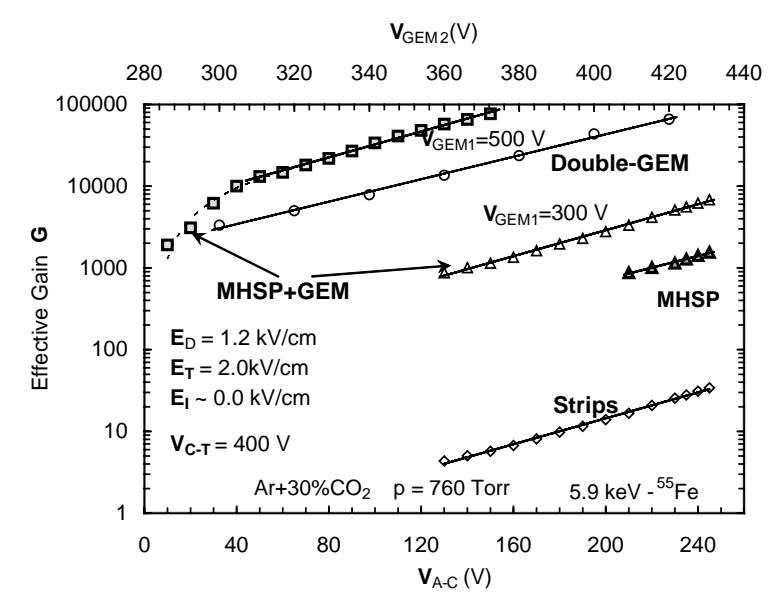

Fig. 3. Gain-voltage characteristics of a MHSP, MHSP + GEM, MHSP strips and double-GEM in $\mathrm{Ar}+30 \% \mathrm{CO}_{2}$ mixture.

absorbed in the transfer gap. A maximum effective gain of $\sim 1.6 \times 10^{3}$ was obtained. By biasing the anode and cathode strips to the same voltage, we could operate the MHSP in a GEM mode and obtain an estimate of the effective gain in the MHSP holes alone; it is of about 50 for a potential difference, $V_{\mathrm{C}-\mathrm{T}}=400 \mathrm{~V}$. Thus, assuming all avalanche electrons are collected on the anode strips, a maximal gain of $\sim 30$ is attributed to the strips, at $V_{\mathrm{A}-\mathrm{C}}=245 \mathrm{~V}$. The estimated gain curve for the strips is also presented in Fig. 3. It should be stressed that, unfortunately, due to misalignment during the manufacturing process, between the MS-pattern and the holes on the MS-side, some of the holes were placed right at the edge of the cathode strip (see Fig. 1). This defect severely limited the maximum applicable voltage difference to about 400 and $245 \mathrm{~V}$ in the MHSP holes and in the strips, respectively. This limitation is associated with spontaneous field emission of electrons from the cathode edges, which led to discharges. For the MHSP + GEM detector we have obtained a maximum effective gain of $\sim 10^{5}$. Higher gains are expected with improved devices.

Fig. 4 shows a pulse-height distribution of the MHSP + GEM detector at the maximum achievable MHSP gain. The energy resolution for the $5.9 \mathrm{keV} \mathrm{X}$-rays peak is about $23 \% \mathrm{FWHM}$, and is similar to that of the double-GEM. For the MHSP alone, the energy resolution is about 29\% FWHM. 


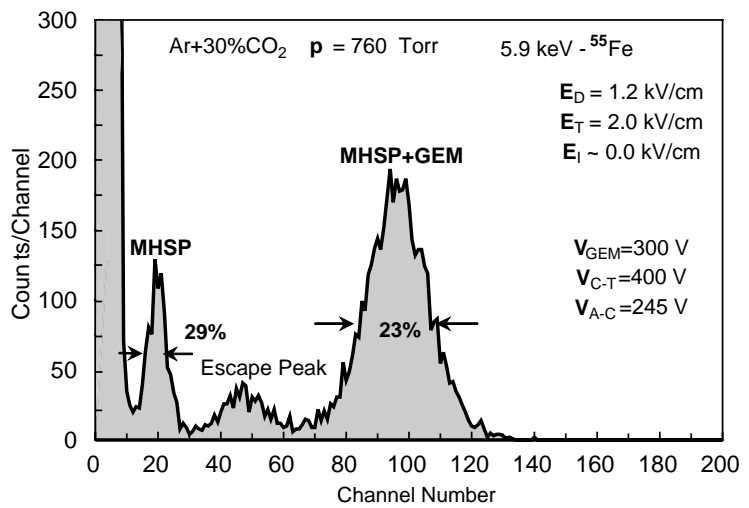

Fig. 4. Pulse-height distribution of $5.9 \mathrm{keV}$ X-rays for MHSP + GEM detector, at maximum MHSP gain, in $\mathrm{Ar}+30 \% \mathrm{CO}_{2}$ mixture.

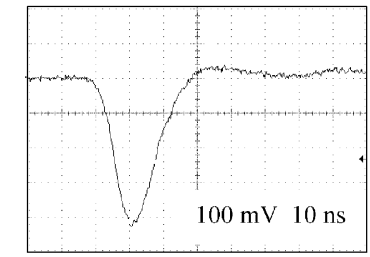

(a)

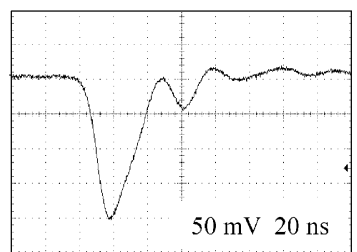

(c)

Fig. 5. Current anode pulses measured in MHSP anode for MHSP + GEM detector (a, b), and in GEM2 bottom for double-GEM detector (c, d). Fast amplifier with $0.5 \mathrm{~ns}$ risetime: (a) and (c); and fast amplifier with 9 ns rise-time: (b) and (d).

Characteristic anode-strip signals from the MHSP + GEM detector, measured with the two current amplifiers at gains close to the maximum values, are presented in Fig. 5a and b. In Fig. 5c and $\mathrm{d}$ we show the GEM2 bottom signals from the double-GEM detector for the same conditions. The fast current amplifier ( $0.5 \mathrm{~ns}$ rise-time) provides quasi-symmetric signals of $6 \mathrm{~ns}$ rise time and $12 \mathrm{~ns}$ pulse-width for the MHSP + GEM detector
(Fig. 5a) and of 8 and $20 \mathrm{~ns}$ for the double-GEM detector (Fig. 5c). A careful inspection of signals from the MHSP + GEM detector shows a small tail, with a 10-time smaller amplitude compared to the main component, extending to $\sim 180-200 \mathrm{~ns}$.

\section{Summary}

We demonstrated the operation principle of compact, micro-pattern gaseous detector, comprising two multiplication steps in a single doublesided electrode. We have shown that indeed two independent multiplication steps occur, and a total gain comparable to that of a single GEM has already been obtained. Moreover, the new detector is expected to be faster than a GEM, due to the fast ion collection on the nearby cathode strips; the potential high-rate capability of the MHSP will be systematically studied in the future. The MHSPs tested here were limited in total gain by their manufacturing shortcoming, and could only be studied in combination with a preamplifying GEM. However, such an operation mode could in general be adopted for improved stability and gain.

A possible application of the MHSP as a photosensor, with a photocathode, e.g. CsI, deposited on its topside is under study. It is a similar principle to that recently demonstrated with a photocathode deposited on GEM [8]. Preliminary simulations indicate that the electric fields at the photocathode surface are sufficiently high to allow for an efficient photoelectron extraction. For single photon detection applications, a photocathode-coated GEM precedes the MHSP to permit higher gains.

The MHSP is expected to have some advantages in minimizing ion-feedback and eliminating photon feedback, similarly to multi-GEM detectors [8].

\section{Acknowledgements}

We would like to thank R. de Oliveira for his help in developing the MHSP, G. Guedes for his cooperation and helpful discussions and M. Klin 
for his technical assistance. The work was partially supported by the project CERN/P/FIS/43785/01 and by the Israel Science Foundation. A. Breskin is the W.P. Reuther Professor of Research in peaceful use of Atomic Energy.

\section{References}

[1] J.F.C.A. Veloso, J.M.F. dos Santos, C.A.N. Conde, Rev. Sci. Instrum. 71 (2000) 2371.
[2] J.M. Maia, J.F.C.A. Veloso, R.E. Morgado, J.M.F. dos Santos, C.A.N. Conde, IEEE Trans. Nucl. Sci. NS-49 (3) (2002) 875.

[3] F. Sauli, Nucl. Instr. and Meth. A 386 (1997) 531.

[4] A. Oed, Nuc. Instr. Meth. A 263 (1988) 351.

[5] R. Bouclier, et al., Nucl. Instr. and Meth. A 396 (1997) 50.

[6] J.M.F. dos Santos, J.A.M. Lopes, J.F.C.A. Veloso, et al., X-ray Spectrom. 30 (2001) 373.

[7] A. Buzulutskov, A. Breskin, G. Garty, R. Chechik, F. Sauli, L. Shekhtmann, Nucl. Instr. and Meth. A 443 (2000) 164.

[8] D. Mörmann, A. Breskin, R. Chechik, P. Cwetanski, B.K. Singh, Nucl. Instr. and Meth. A 478 (2002) 230. 